\title{
TENSOR PRODUCTS OF MEASURABLE OPERATORS
}

\author{
M. ANOUSSIS, V. FELOUZIS AND I. G. TODOROV
}

\begin{abstract}
We introduce and study a stability property for submodules of measurable operators and Calkin spaces and characterize the tensor stable singly generated Calkin spaces. Given semifinite von Neumann algebras $(\mathcal{M}, \tau),(\mathcal{N}, \sigma)$ and corresponding measurable operators $S, T$, we provide a necessary and sufficient condition for the operator $S \otimes T$ to be measurable with respect to $(\mathcal{M} \otimes \mathcal{N}, \tau \otimes \sigma)$.
\end{abstract}

\section{Introduction}

Weiss considered in [14] a property for ideals of $\mathbf{B}(\mathcal{H})$, called "tensor product closure property", or "tensor stability". In a previous paper [1], we studied the analogous property for Calkin sequence spaces. In particular, we established a necessary and sufficient condition for the tensor stability of a singly generated Calkin sequence space.

In this paper, we study the analogous stability property for submodules of measurable operators and Calkin function spaces. Using results of O'Neil, we describe a large class of stable submodules of measurable operators. We then focus on singly generated Calkin function spaces. We give a necessary and sufficient condition for the tensor stability of a singly generated Calkin function space and provide examples of stable singly generated Calkin function spaces.

Let $(\mathcal{M}, \tau),(\mathcal{N}, \sigma)$ be two semifinite von Neumann algebras and $S, T$ measurable operators with respect to $(\mathcal{M}, \tau),(\mathcal{N}, \sigma)$. In the first part of the paper, we give a necessary and sufficient condition for $S \otimes T$ to be a $\tau \otimes \sigma$ measurable operator. This characterization is used in an essential way in the study of stable submodules.

We now introduce some notation. If $\mathcal{H}$ is a Hilbert space, we denote by $\mathbf{B}(\mathcal{H})$ the algebra of bounded linear operators on $\mathcal{H}$. If $X$ is a set and $A \subseteq$

Received October 22, 2015; received in final form March 7, 2016.

2010 Mathematics Subject Classification. Primary 46L10. Secondary 46L52. 
$X$, we denote by $\chi_{A}$ the characteristic function of $A$. By $m$ we denote the Lebesgue measure. If $\mathcal{M}$ is a von Neumann algebra, and $P \in \mathcal{M}$ we say that $P$ is a projection if $P$ is a selfadjoint idempotent.

\section{The algebra $\overline{\mathcal{M}}$}

Let $\mathcal{M}$ be a von Neumann algebra acting on a Hilbert space $\mathcal{H}$. Let $\widetilde{\mathcal{M}}$ be the set of all operators $T$ in $\mathcal{H}$ which are densely defined, closed and affiliated to $\mathcal{M}$.

Assume that $\mathcal{M}$ admits a faithful semi-finite normal trace $\tau$. Let $\overline{\mathcal{M}}$ be the subset of $\widetilde{\mathcal{M}}$ consisting of all $T \in \widetilde{\mathcal{M}}$ such that $\lim _{s \rightarrow+\infty} \tau\left(E^{|T|}(s,+\infty)\right)=$ 0 , where $|T|=\left(T^{*} T\right)^{1 / 2}$ and $E^{|T|}(s,+\infty)$ is the spectral projection of $|T|$ corresponding to the interval $(s,+\infty)$. Then $\overline{\mathcal{M}}$ is a $*$-algebra with respect to the operations $(S, T) \mapsto \overline{S+T},(S, T) \mapsto \overline{S T}, T \mapsto T^{*}$, where $\bar{T}$ denotes the closure of an operator $T$ [5, Paragraph 1.4]. The operators in $\overline{\mathcal{M}}$ are called $\tau$-measurable. In the sequel, we shall write $S+T$ instead of $\overline{S+T}$ and $S T$ instead of $\overline{S T}$.

On $\overline{\mathcal{M}}$, we consider the measure topology, introduced in [10], which is the translation invariant topology defined by the neighborhoods of 0 of the form

$$
\begin{aligned}
U_{\varepsilon, \delta}= & \{T: \text { there exits a projection } P \in \mathcal{M} \\
& \text { such that } \left.\|T P\|<\varepsilon \text { and } \tau\left(P^{\perp}\right)<\delta\right\},
\end{aligned}
$$

where $\varepsilon, \delta>0$ and, for a projection $P$, we have set $P^{\perp}=I-P$. Then $\overline{\mathcal{M}}$ becomes a complete topological $*$-algebra and $\mathcal{M}$ is a dense $*$-subalgebra of $\overline{\mathcal{M}}[10]$.

Let $T \in \overline{\mathcal{M}}$ and $t>0$. We set

$$
\mu_{t}(T)=\inf \{\|T P\|: P \text { is a projection in } \mathcal{M} \text { and } \tau(1-P) \leq t\} .
$$

We will denote the function $t \rightarrow \mu_{t}(T)$ by $\mu(T)$.

We collect some properties of the function $\mu(T)$ in the following proposition [5, Proposition 2.2, Lemmata 2.5, 2.6].

Proposition 2.1. Let $T, S, R$ be $\tau$-measurable operators. Then the following properties are satisfied:

(i) The map $t \rightarrow \mu_{t}(T)$ from $(0,+\infty)$ to $[0,+\infty]$ is non-increasing and rightcontinuous. Moreover,

$$
\lim _{t \rightarrow 0} \mu_{t}(T)=\|T\| \in[0,+\infty] .
$$

(ii) $\mu_{t}(T)=\mu_{t}(|T|)=\mu_{t}\left(T^{*}\right)$, for $t>0$.

(iii) $\mu_{t}(T)=\inf \left\{s \geq 0: \tau\left(E^{|T|}(s,+\infty)\right) \leq t\right\}$.

(iv) $\mu_{t+s}(T+S) \leq \mu_{t}(T)+\mu_{s}(S)$, for $t>0, s>0$.

(v) $\mu_{t}(R T S) \leq\|R\|\|S\| \mu_{t}(T), t>0$.

(vi) For every $t>0$ and for every projection $P \in \mathcal{M}$ with $\tau(P) \leq t$ we have that $\mu_{t}(T P)=0$. 
(vii) For every $t>0$ and for every projection $P \in \mathcal{M}$ with $\tau(P)>t$ we have that $\mu_{t}(P)=1$.

Let $T$ in $\overline{\mathcal{M}}$. We will say that $T$ is a $\tau$-finite-rank operator if there exists a projection $P \in \mathcal{M}$ such that $\tau(P)<+\infty$ and $T=P T$. It follows from Proposition 2.1(ii), (iii) and (vi) that $T$ is a $\tau$-finite rank operator if and only if $m(\operatorname{supp}(\mu(T)))<+\infty$. It follows from Proposition 2.1(i) that $T$ is bounded if and only if $\mu(T)$ is bounded.

\section{Tensor admissibility}

Let $(X, \nu)$ be a $\sigma$-finite measure space. Let us denote by $\mathscr{M}(X)$ the linear space of all $\nu$-measurable functions $f: X \rightarrow \mathbb{C}$, where we identify the functions which are equal $\nu$-a.e. Let $f \in \mathscr{M}(X)$. The distribution function of $f$ is the function $\delta_{f}:(0,+\infty) \rightarrow[0,+\infty]$ given by

$$
\delta_{f}(s)=\nu(\{x \in X:|f(x)|>s\}) .
$$

It is trivial to verify that $\delta_{f}$ is a non-increasing right-continuous function. The decreasing rearrangement of $f$ is the function $f^{\star}:(0,+\infty) \rightarrow[0,+\infty]$ given by:

$$
f^{\star}(t)=\inf \left\{s \geq 0: \delta_{f}(s) \leq t\right\} .
$$

Note that, since $\delta_{f}$ is right continuous, the latter infimum is attained, and that $f^{\star}$ is right continuous [2, Chapter 2, Proposition 1.7].

The following are equivalent for a function $f \in \mathscr{M}(X)$ :

(1) there exists a $t>0$ such that $\delta_{f}(t)<+\infty$,

(2) $\lim _{t \rightarrow+\infty} \delta_{f}(t)=0$,

(3) $f^{\star}(t) \neq+\infty$ for every $t>0$.

We call a function $f \in \mathscr{M}(X)$ admissible if $f$ satisfies the above conditions. The set of all admissible functions is a subspace of $\mathscr{M}(X)$ [2, Chapter 2, Proposition 1.3] which we will denote by $\mathscr{L}(X)$.

We equip the set $(0,+\infty)$ with the Lebesgue measure $m$ and set $\mathscr{M}=$ $\mathscr{M}((0,+\infty))$ and $\mathscr{L}=\mathscr{L}((0,+\infty))$.

Let $L^{\infty}(m)$ be the von Neumann algebra of all $m$-measurable essentially bounded functions $f:(0,+\infty) \rightarrow \mathbb{C}$. Then the map $\tau: L^{\infty}(m) \rightarrow \mathbb{C}$ defined by $\tau(f)=\int f d m$ is a semifinite normal trace on $L^{\infty}(m)$. The space $\widehat{L^{\infty}(m)}$ of operators affiliated to $L^{\infty}(m)$ is $\mathscr{M}$, while the space of $\tau$-measurable operators $\overline{L^{\infty}(m)}$ coincides with $\mathscr{L}$ and $\mu(f)=f^{*}$ for every $f \in \mathscr{L}$.

We will denote by $\mathscr{D}$ the cone of all decreasing and right continuous functions $f:(0,+\infty) \rightarrow[0,+\infty)$. Note that $\mathscr{L}=\left\{f \in \mathscr{M}: f^{*} \in \mathscr{D}\right\}$.

Let $f, g \in \mathscr{L}$. We denote by $f \otimes g$ the function $f \otimes g:(0,+\infty) \times(0,+\infty) \rightarrow$ $\mathbb{C}$ defined by

$$
(f \otimes g)(x, y)=f(x) g(y) .
$$

Definition 3.1. (1) A pair $(f, g) \in \mathscr{L} \times \mathscr{L}$ is called tensor admissible if $(f \otimes g)^{\star} \in \mathscr{D}$. 
(2) A function $f \in \mathscr{L}$ is called tensor admissible if the pair $(f, f)$ is tensor admissible.

Note that, by definition, a pair $(f, g)$ is tensor admissible if and only if $(f \otimes g)^{\star}(t)<+\infty$ for every $t>0$.

Definition 3.2. Let $f \in \mathscr{D}$ and $\theta \in(0,1)$. For $n \in \mathbb{Z}$ we set

$$
\begin{aligned}
I_{n}(f, \theta) & =\left\{t>0: \theta^{n+1}<f(t) \leq \theta^{n}\right\}, \quad J_{n}(f, \theta)=\bigcup_{i<n} I_{i}(f, \theta), \\
a_{n}(f, \theta) & =m\left(I_{n}(f, \theta)\right), \quad A_{n}(f, \theta)=\sum_{i<n} a_{i}(f, \theta)=m\left(J_{n}(f, \theta)\right), \\
L_{\theta}(f) & =\sum_{n \in \mathbb{Z}} \theta^{n} \chi_{I_{n}(f, \theta) .}
\end{aligned}
$$

We call the function $L_{\theta}(f)$ the $\theta$-approximation of $f$.

Let $f$ and $g$ be two real valued functions in $\mathscr{L}$. We shall write $f \lesssim g$ if there exists a constant $C>0$ such that for every $x \in(0,+\infty)$ we have $f(x) \leq C g(x)$. If $f \lesssim g$ and $g \lesssim f$ we will say that $f$ and $g$ are equivalent and we will write $f \sim g$.

The following remark follows directly from the definitions.

REMARK 3.3. Let $f \in \mathscr{D}$ and $\theta \in(0,1)$. Then

(1) $I_{n}(f, \theta)=\left[A_{n}(f, \theta), A_{n+1}(f, \theta)\right)=\left[A_{n}(f, \theta), A_{n}(f, \theta)+a_{n}(f, \theta)\right)$.

(2) The $\theta$-approximation $L_{\theta}(f)$ of $f$ is decreasing and right continuous and hence belongs to $\mathscr{D}$.

(3) The $\theta$-approximation $L_{\theta}(f)$ of $f$ satisfies $\theta L_{\theta}(f) \leq f \leq L_{\theta}(f)$. Thus, $f \sim$ $L_{\theta}(f)$.

(4) We have $I_{n}(f, \theta)=I_{n}\left(L_{\theta}(f), \theta\right)$ and $a_{n}(f, \theta)=a_{n}\left(L_{\theta}(f), \theta\right)$ for every $n \in \mathbb{Z}$.

(5) $m(\operatorname{supp} f)<+\infty$ if and only if $\sum_{n \in \mathbb{Z}} a_{n}(f, \theta)<+\infty$.

(6) $f$ is bounded if and only if there exists $n_{0}$ such that $a_{n}(f, \theta)=0$ for $n \leq n_{0}$.

(7) $L_{\theta}\left(L_{\theta}(f)\right)=L_{\theta}(f)$.

The proof of the following lemma is straightforward and we omit it.

LEMMA 3.4. Let $f, g, f^{\prime}, g^{\prime} \in \mathscr{D}$. If $f \lesssim f^{\prime}$ and $g \lesssim g^{\prime}$ then $(f \otimes g)^{\star} \lesssim$ $\left(f^{\prime} \otimes g^{\prime}\right)^{\star}$.

In the sequel, we use the conventions $0 \cdot(+\infty)=0$ and $[+\infty,+\infty)=\emptyset$.

TheOREM 3.5. Let $f, g$ be in $\mathscr{D}$ and $\theta \in(0,1)$. Let $a_{n}=a_{n}(f, \theta)$ and $b_{n}=a_{n}(g, \theta), n \in \mathbb{Z}$. For every $k \in \mathbb{Z}$, we set $r_{k}=\sum_{i+j<k} a_{i} b_{j}$. Then the pair $(f, g)$ is tensor admissible if and only if there exists $k_{0}$ such that $r_{k_{0}}<+\infty$. In that case, we have that

$$
(f \otimes g)^{\star} \sim \sum_{k \in \mathbb{Z}} \theta^{k} \chi_{\left[r_{k}, r_{k+1}\right)} .
$$


Proof. It follows from Lemma 3.4 and Remark 3.3 that the pair $(f, g)$ is tensor admissible if and only if the pair $\left(L_{\theta}(f), L_{\theta}(g)\right)$ is tensor admissible and also that $a_{n}(f, \theta)=a_{n}\left(L_{\theta}(f), \theta\right)$ and $a_{n}(g, \theta)=a_{n}\left(L_{\theta}(g), \theta\right)$. By $(7)$ above, we may suppose that $f=L_{\theta}(f)$ and $g=L_{\theta}(g)$.

Let $t>0$.

Then $f(x) g(y)>t$ if and only if there exist $i, j$ such that $x \in I_{i}(f, \theta)$, $y \in I_{j}(g, \theta)$ and $\theta^{i} \theta^{j}=\theta^{i+j}>t$. Therefore,

$$
\{(x, y): f(x) g(y)>t\}=\bigcup_{i, j, \theta^{i+j}>t}\left\{(x, y): x \in I_{i}(f, \theta), y \in I_{j}(g, \theta)\right\} .
$$

Let $k \in \mathbb{Z}$ be such that $\theta^{k} \leq t<\theta^{k-1}$. We have

$$
\begin{aligned}
& \bigcup_{i, j, \theta^{i+j}>t}\left\{(x, y): x \in I_{i}(f, \theta), y \in I_{j}(g, \theta)\right\} \\
& =\bigcup_{i, j, \theta^{i+j}>\theta^{k}}\left\{(x, y): x \in I_{i}(f, \theta), y \in I_{j}(g, \theta)\right\} \\
& =\bigcup_{i, j, i+j<k}\left\{(x, y): x \in I_{i}(f, \theta), y \in I_{j}(g, \theta)\right\} .
\end{aligned}
$$

Thus

$$
\begin{aligned}
\delta_{f \otimes g}(t) & =m(\{(x, y): f(x) g(y)>t\}) \\
& =m\left(\bigcup_{i, j, i+j<k}\left\{(x, y): x \in I_{i}(f, \theta), y \in I_{j}(g, \theta)\right\}\right)=r_{k} .
\end{aligned}
$$

The pair $(f, g)$ is tensor admissible if and only if $\delta_{f \otimes g}(t)<+\infty$ for some $t$. Hence, the pair $(f, g)$ is tensor admissible if and only if for some $k \in \mathbb{Z}, r_{k}<$ $+\infty$. The proof of the first assertion is complete.

Let $k \in \mathbb{Z}$ be such that $r_{k} \leq s<r_{k+1}$. Let $\varepsilon>0$ be such that $\theta^{k}+\varepsilon<\theta^{k-1}$. By the first part of the proof it follows that $\delta_{f \otimes g}\left(\theta^{k}+\varepsilon\right)=r_{k} \leq s$ which implies that $(f \otimes g)^{*}(s) \leq \theta^{k}+\varepsilon$. Let $\varepsilon>0$ be such that $\theta^{k}-\varepsilon>\theta^{k+1}$. Again by the first part of the proof we have that $\delta_{f \otimes g}\left(\theta^{k}-\varepsilon\right)=r_{k+1}>s$, which implies that $(f \otimes g)^{*}(s) \geq \theta^{k}-\varepsilon$. Hence, $(f \otimes g)^{*}(s)=\theta^{k}$.

The proof of the following corollary is contained in the proof of Theorem 3.5.

Corollary 3.6. Let $f, g$ be in $\mathscr{D}$ and $\theta \in(0,1)$. Assume that $f=L_{\theta}(f)$ and that $g=L_{\theta}(g)$. Let $a_{n}=a_{n}(f, \theta), b_{n}=a_{n}(g, \theta)$. For every $k \in \mathbb{Z}$, we set $r_{k}=\sum_{i+j<k} a_{i} b_{j}$. Then

$$
(f \otimes g)^{\star}=\sum_{k \in \mathbb{Z}} \theta^{k} \chi_{\left[r_{k}, r_{k+1}\right)} .
$$


(2)

$$
a_{n}\left((f \otimes g)^{*}\right)=\sum_{i+j=n} a_{i} b_{j}
$$

for every $n \in \mathbb{Z}$.

Proposition 3.7. Let $f \in \mathscr{D}$. If $f$ is unbounded and tensor admissible, then $\lim _{x \rightarrow+\infty} f(x)=0$.

Proof. Assume $\lim _{x \rightarrow+\infty} f(x)=a \neq 0$. Let $r>0$. Since $f$ is unbounded the set $A=\{t: f(t)>r / a\}$ has positive measure. Then $\delta_{f \otimes f}(r)=(m \times m)(\{(t, s)$ : $|f(t) f(s)|>r\}) \geq(m \times m)(\{(t, s): t \in A, s \in(0,+\infty)\})=+\infty$.

All the von Neumann algebras, we consider from now on, are semifinite and atomless. Let $\mathcal{H}_{1}, \mathcal{H}_{2}$ be Hilbert spaces and $\mathcal{M} \subseteq B\left(\mathcal{H}_{1}\right)$ and $\mathcal{N} \subseteq B\left(\mathcal{H}_{2}\right)$ be von Neumann algebras. Assume that $\tau$ (resp. $\sigma$ ) is a faithful semi-finite normal trace on $\mathcal{M}$ (resp. $\mathcal{N}$ ).

Let $\mathcal{H}=\mathcal{H}_{1} \otimes \mathcal{H}_{2}$ be the Hilbert tensor product of $\mathcal{H}_{1}$ and $\mathcal{H}_{2}$. We denote by $(\mathcal{M} \otimes \mathcal{N}, \tau \otimes \sigma)$ the spatial tensor product of $(\mathcal{M}, \tau)$ and $(\mathcal{N}, \sigma)$, that is, the von Neumann algebra acting on $\mathcal{H}_{1} \otimes \mathcal{H}_{2}$, generated by the operators $A \otimes B$, where $A \in \mathcal{M}$ and $B \in \mathcal{N}$, equipped with the trace $\tau \otimes \sigma$ defined by $(\tau \otimes \sigma)(A \otimes B)=\tau(A) \sigma(B), A \in \mathcal{M}, B \in \mathcal{N}$. If $A \in \overline{\mathcal{M}}$ and $B \in \overline{\mathcal{N}}$ then $A \otimes B$ is a closed, densely defined operator affiliated to $\mathcal{M} \bar{\otimes} \mathcal{N}$ and $(A \otimes B)^{*}=$ $A^{*} \otimes B^{*}[12$, Theorem 8.1], but it is not true in general that $A \otimes B \in \overline{\mathcal{M}} \bar{\otimes} \mathcal{N}$. The next theorem provides a characterization of the pairs $(A, B) \in \overline{\mathcal{M}} \times \overline{\mathcal{N}}$ with the property that $A \otimes B \in \overline{\mathcal{M} \bar{\otimes} \mathcal{N}}$.

TheOREM 3.8. Let $\mathcal{H}_{1}$ (resp. $\left.\mathcal{H}_{2}\right)$ be a Hilbert space, and $\mathcal{M}$ (resp. $\left.\mathcal{N}\right)$ be a Neumann algebra equipped with a faithful semi-finite normal trace $\tau$ (resp. $\sigma$ ). Let $A \in \overline{\mathcal{M}}$ and $B \in \overline{\mathcal{N}}$. Then $A \otimes B \in \overline{\mathcal{M} \bar{\otimes} \mathcal{N}}$ if and only if the pair $(\mu(A), \mu(B))$ is tensor admissible. In this case, we have that

$$
\mu(A \otimes B)=(\mu(A) \otimes \mu(B))^{\star} .
$$

Proof. Using polar decomposition, we may suppose that $A, B$ are positive operators. Let $E^{A}$ (resp. $E^{B}$ ) be the spectral measure of $A$ (resp. $B$ ); thus, $A=\int_{0}^{+\infty} x d E^{A}(x)$ and $B=\int_{0}^{+\infty} y d E^{B}(y)$. Let $E^{A} \otimes E^{B}$ be the spectral measure on $\mathbb{R} \times \mathbb{R}$ with values in the projection lattice of $\mathcal{H}_{1} \otimes \mathcal{H}_{2}$ defined by

$$
E^{A} \otimes E^{B}\left(\delta_{1} \times \delta_{2}\right)=E^{A}\left(\delta_{1}\right) \otimes E^{B}\left(\delta_{2}\right),
$$

where $\delta_{1}, \delta_{2}$ are Borel subsets of $\mathbb{R}$. It follows from [12, Theorem 8.2] that

$$
A \otimes B=\int_{0}^{+\infty} \int_{0}^{+\infty} x y d\left(E^{A} \otimes E^{B}\right)(x, y) .
$$

Let us denote by $E^{A, B}$ the spectral measure on $\mathbb{R}$ given by

$$
E^{A, B}(\delta)=\left(E^{A} \otimes E^{B}\right)(\{(x, y): x y \in \delta\}) .
$$


Then

$$
A \otimes B=\int_{0}^{+\infty} x d E^{A, B}(x) .
$$

For every $s>0$, we set

$$
\Delta_{s}=\{(x, y) \in(0,+\infty) \times(0,+\infty): x y>s\} ;
$$

note that $E^{A, B}(s,+\infty)=\left(E^{A} \otimes E^{B}\right)\left(\Delta_{s}\right)$.

Note that $A \otimes B \in \overline{\mathcal{M}} \bar{\otimes} \mathcal{N}$ if and only if for every $t>0$ we have that $\mu_{t}(A \otimes B)<+\infty$. Since

$$
\mu_{t}(A \otimes B)=\inf \left\{s \geq 0: \tau \otimes \sigma\left(E^{A} \otimes E^{B}\left(\Delta_{s}\right)\right) \leq t\right\}
$$

and

$$
(\mu(A) \otimes \mu(B))^{\star}(t)=\inf \left\{s \geq 0:(m \times m)\left(\left\{(x, y): \mu_{x}(A) \mu_{y}(B)>s\right\}\right) \leq t\right\}
$$

the conclusion of the theorem will follow if we prove the following equality:

$$
(\tau \otimes \sigma)\left(E^{A} \otimes E^{B}\left(\Delta_{s}\right)\right)=(m \times m)\left(\left\{(x, y): \mu_{x}(A) \mu_{y}(B)>s\right\}\right) .
$$

Let $s>0$. For every $i, n \in \mathbb{N}$, set

$$
\begin{array}{ll}
I_{(n, i)} & =\left[\frac{i}{n}, \frac{i+1}{n}\right), \quad J_{(n, i)}=\left(\frac{s}{i / n},+\infty\right), \\
\delta_{(n, i)} & =I_{(n, i)} \times J_{(n, i)}, \quad \text { and } \quad \delta_{n}=\bigcup_{i=1}^{+\infty} \delta_{(n, i)} .
\end{array}
$$

Clearly,

$$
\delta_{1} \subseteq \delta_{2} \subseteq \delta_{3} \subseteq \cdots \quad \text { and } \quad \Delta_{s}=\bigcup_{n=1}^{+\infty} \delta_{n} .
$$

By [5, Remark 3.3], for every Borel subset $\delta$ of $\mathbb{R}$ and every positive operator $T \in \overline{\mathcal{M}}$ we have that

$$
\tau\left(E^{T}(\delta)\right)=\int_{0}^{+\infty} \chi_{\delta}\left(\mu_{t}(T)\right) d t=m\left(\left\{x: \mu_{x}(T) \in \delta\right\}\right) .
$$

By (4) and (5),

$$
\begin{aligned}
(\tau & \otimes \sigma)\left(E^{A} \otimes E^{B}\left(\Delta_{s}\right)\right) \\
& =\lim _{n \rightarrow \infty}(\tau \otimes \sigma)\left(E^{A} \otimes E^{B}\left(\delta_{n}\right)\right) \\
& =\lim _{n \rightarrow \infty} \sum_{i=1}^{+\infty} \tau \otimes \sigma\left(E^{A} \otimes E^{B}\left(\delta_{(n, i)}\right)\right) \\
& =\lim _{n \rightarrow \infty} \sum_{i=1}^{+\infty} \tau\left(E^{A}\left(I_{(n, i)}\right)\right) \sigma\left(E^{B}\left(J_{(n, i)}\right)\right)
\end{aligned}
$$




$$
\begin{aligned}
& =\lim _{n \rightarrow \infty} \sum_{i=1}^{+\infty} m\left(\left\{x: \mu_{x}(A) \in I_{(n, i)}\right\}\right) m\left(\left\{y: \mu_{y}(B) \in J_{(n, i)}\right\}\right) \\
& =\lim _{n \rightarrow \infty} \sum_{i=1}^{+\infty}(m \times m)\left(\left\{x: \mu_{x}(A) \in I_{(n, i)}\right\} \times\left\{y: \mu_{y}(B) \in J_{(n, i)}\right\}\right) \\
& =\lim _{n \rightarrow \infty}(m \times m)\left(\bigcup_{i=1}^{+\infty}\left\{(x, y): \mu_{x}(A) \in I_{(n, i)}, \mu_{y}(B) \in J_{(n, i)}\right\}\right) \\
& =(m \times m)\left(\bigcup_{n=1}^{+\infty} \bigcup_{i=1}^{+\infty}\left\{(x, y): \mu_{x}(A) \in I_{(n, i)}, \mu_{y}(B) \in J_{(n, i)}\right\}\right) \\
& =(m \times m)\left(\left\{(x, y): \mu_{x}(A) \mu_{y}(B)>s\right\}\right),
\end{aligned}
$$

and (3) is established.

Recall that $L^{\infty}(m)$ is the von Neumann algebra of all $m$-measurable essentially bounded functions $f:(0,+\infty) \rightarrow \mathbb{C}$ equipped with the faithful semifinite normal trace $\tau$ given by $\tau(f)=\int f d m$. We also have that $\mathscr{L}=\overline{L^{\infty}(m)}$. Setting $\mathcal{M}=\mathcal{N}=L^{\infty}(m)$ in the above theorem we obtain the following.

Corollary 3.9. Let $f \in \mathscr{L}$. If $f$ is tensor admissible, then

$$
(f \otimes f)^{\star}=\left(f^{\star} \otimes f^{\star}\right)^{\star} .
$$

Definition 3.10. Let $(\mathcal{M}, \tau),(\mathcal{N}, \sigma)$ be von Neumann algebras with faithful semi-finite normal traces $\tau, \sigma$. Let $T \in \overline{\mathcal{M}}, S \in \overline{\mathcal{N}}$.

(1) We will call the pair $(T, S)$ tensor admissible if $T \otimes S \in \overline{\mathcal{M}} \bar{\otimes} \mathcal{N}$.

(2) We will call $T$ tensor admissible if $T \otimes T \in \overline{\mathcal{M} \otimes \mathcal{M}}$.

REMARK 3.11. By Theorem 3.8, the pair $(S, T)$ (resp. the operator $T$ ) is tensor admissible if and only if the pair $(\mu(S), \mu(T))$ (resp. the function $\mu(T)$ ) is tensor admissible.

Some properties of tensor admissible operators are described in the following theorem.

THEOREM 3.12. Let $(\mathcal{M}, \tau)$ be a von Neumann algebra with a faithful semifinite normal trace $\tau$. Let $S, T \in \overline{\mathcal{M}}$.

(1) If $S$ and $T$ are bounded, then the pair $(S, T)$ is tensor admissible.

(2) If $S$ and $T$ are $\tau$-finite-rank operators, then the pair $(S, T)$ is tensor admissible.

(3) If $T$ is tensor admissible and $S$ is a bounded $\tau$-finite-rank operator, then $T+S$ is tensor admissible.

Proof. (1) is clear.

(2) It suffices to show that the pair $(\mu(S), \mu(T))$ is tensor admissible. Since $S$ and $T$ are $\tau$-finite-rank operators, there exists $r_{0}$ such that $\mu_{r_{0}}(S)=0$ and 
$\mu_{r_{0}}(T)=0$. The pair $(\mu(S), \mu(T))$ is tensor admissible if, for some $r>0$, the set $\left\{(x, y): \mu_{x}(S) \mu_{y}(T)>r\right\}$ has finite Lebesgue measure. Since

$$
\left\{(x, y): \mu_{x}(S) \mu_{y}(T)>r\right\} \subseteq\left\{(x, y): \mu_{x}(S) \mu_{y}(T)>0\right\}
$$

and $(m \times m)\left(\left\{(x, y): \mu_{x}(S) \mu_{y}(T)>0\right\}\right) \leq r_{0}^{2}$, the assertion follows.

(3) If $T$ is a $\tau$-finite-rank operator, the assertion follows from (2). We assume that $T$ is not a $\tau$-finite-rank operator. Let $s_{0}$ be such that $\mu_{s_{0}}(S)=0$. Since $T$ is tensor admissible, there exists $r$ such that the measure of the set

$$
F=\left\{(x, y): \mu_{x}(T) \mu_{y}(T)>r\right\}
$$

is finite. By Proposition 2.1, for $x \leq s_{0}$ we have that

$$
\mu_{x}(T+S) \leq \mu_{x}(T)+\|S\| .
$$

Set $c=\frac{\|S\|}{\mu_{s_{0}}(T)}$ (note that, since $T$ is not $\tau$-finite-rank, $\mu_{s_{0}}(T)>0$ ). We have

$$
\frac{\mu_{x}(T+S)}{\mu_{x}(T)} \leq \frac{\mu_{x}(T)+\|S\|}{\mu_{x}(T)} \leq 1+\frac{\|S\|}{\mu_{x}(T)} \leq 1+\frac{\|S\|}{\mu_{s_{0}}(T)}=1+c .
$$

It follows that

$$
\mu_{x}(T+S) \leq \mu_{x}(T)(1+c)
$$

if $0<x \leq s_{0}$.

For every $x>s_{0}$, by Proposition 2.1 we have that

$$
\mu_{x}(T+S) \leq \mu_{x-s_{0}}(T)+\mu_{s_{0}}(S)=\mu_{x-s_{0}}(T) .
$$

We show that the set

$$
E=\left\{(x, y): \mu_{x}(T+S) \mu_{y}(T+S)>r(1+c)^{2}\right\}
$$

has finite measure. Assume that $x \leq s_{0}, y \leq s_{0}$ and $(x, y) \in E$. Then

$$
\mu_{x}(T+S) \mu_{y}(T+S)>r(1+c)^{2}
$$

and hence, by $(6), \mu_{x}(T) \mu_{y}(T)>r$. Thus, the set

$$
\left\{(x, y) \in E: x \leq s_{0}, y \leq s_{0}\right\}
$$

is contained in $F$ and therefore has finite measure.

Assume $x \leq s_{0}, y>s_{0}$ and $(x, y) \in E$. We have that

$$
\mu_{x}(T+S) \mu_{y}(T+S)>r(1+c)^{2}
$$

and hence, by (6) and (7),

$$
\mu_{x}(T) \mu_{y-s_{0}}(T)>r(1+c)>r .
$$

Set

$$
A=\left\{(x, y): x \leq s_{0}, y>s_{0}, \mu_{x}(T) \mu_{y-s_{0}}(T)>r\right\}
$$

and

$$
B=\left\{(x, y): x \leq s_{0}, y>0, \mu_{x}(T) \mu_{y}(T)>r\right\} .
$$


Then $B$ is contained in $F$ and hence has finite measure; since $A$ is the translate of $B$ by the point $\left(0, s_{0}\right)$, we have that $A$ has finite measure. Since $\{(x, y) \in$ $\left.E: x \leq s_{0}, y>s_{0}\right\}$ is contained in $A$, it has finite measure.

Similarly, we can show that the set $\left\{(x, y) \in E: x>s_{0}, y \leq s_{0}\right\}$ has finite measure.

Assume $x>s_{0}, y>s_{0}$ and $(x, y) \in E$. We have

$$
\mu_{x}(T+S) \mu_{y}(T+S)>r(1+c)^{2}
$$

and hence, by (7),

$$
\mu_{x-s_{0}}(T) \mu_{y-s_{0}}(T)>r(1+c)^{2}>r .
$$

Set

$$
A^{\prime}=\left\{(x, y), x>s_{0}, y>s_{0}: \mu_{x-s_{0}}(T) \mu_{y-s_{0}}(T)>r\right\}
$$

and

$$
B^{\prime}=\left\{(x, y), x>0, y>0: \mu_{x}(T) \mu_{y}(T)>r\right\} .
$$

As before, $B^{\prime}$ has finite measure; since $A^{\prime}$ is the translate of $B^{\prime}$ by the point $\left(s_{0}, s_{0}\right)$, we have that $A^{\prime}$ is of finite measure. As $\left\{(x, y) \in E: x>s_{0}, y>s_{0}\right\}$ is contained in $A^{\prime}$, it has finite measure. It follows that $E$ has finite measure as the union of four sets of finite measure. The statement now follows from Remark 3.11.

The following proposition is [4, Proposition 1 and Lemma 9]. Note that [4, Lemma 9] was formulated for bounded functions $f$, but its proof works in the case $f \in \mathscr{D}$ as well.

Proposition 3.13. Let $\mathcal{M}$ be a type $I I_{\infty}$ factor acting on a separable Hilbert space with a faithful semi-finite normal trace $\tau$. There exists an increasing strongly continuous function $P:[0,+\infty) \rightarrow \mathcal{M}$, denoted $t \mapsto P_{t}$ such that:

(1) For every $t \in[0,+\infty), P_{t}$ is a projection in $\mathcal{M}$.

(2) For every $t \in[0,+\infty)$ we have that $\tau\left(P_{t}\right)=t$.

(3) $\lim _{t \rightarrow \infty} P_{t}=I$.

Moreover, if $f$ is a function in $\mathscr{D}$ and $T=\int_{0}^{+\infty} f(t) d P_{t}$, then $T$ is a measurable operator and $\mu_{t}(T)=f(t)$.

EXAMPLE 3.14 (A measurable non-tensor admissible operator). We will construct a function in $\mathscr{D}$ which is not tensor admissible and then using Proposition 3.13 we will find a measurable non-tensor admissible operator.

Let $a>0$. We consider the function $f:(0,+\infty) \rightarrow[0,+\infty)$ defined by $f(t)=t^{-a}$. We have $I_{n}(f, 1 / 2)=\left[2^{n / a}, 2^{(n+1) / a}\right)$. Set $a_{n}=a_{n}(f, 1 / 2)$; we have

$$
a_{n}=m\left(I_{n}(f, 1 / 2)\right)=2^{n / a}\left(2^{1 / a}-1\right)
$$


It follows from Theorem 3.5 that $f$ is tensor admissible if and only if there exists $k_{0}$ such that $r_{k_{0}}<+\infty$, where $r_{k}=\sum_{i+j<k} a_{i} a_{j}$ for each $k \in \mathbb{Z}$. For $k \in \mathbb{Z}$, we have

$$
\begin{aligned}
r_{k} & \geq \sum_{i+j=k-1} a_{i} a_{j}=\sum_{i+j=k-1} 2^{i / a} 2^{j / a}\left(2^{1 / a}-1\right)^{2} \\
& =\sum_{i \in \mathbb{Z}} 2^{(k-1) / a}\left(2^{1 / a}-1\right)^{2}=+\infty .
\end{aligned}
$$

We conclude that $f$ is not tensor admissible. Let $\mathcal{M}, \tau$ and $P$ be as in Proposition 3.13, and $T=\int_{0}^{+\infty} f(t) d P_{t}$. The operator $T$ is measurable and $\mu_{t}(T)=f(t)$. By Theorem 3.8, $T$ is not tensor admissible.

EXAMPLE 3.15 (Two tensor admissible positive measurable operators whose sum is not tensor admissible). Let $a>0$ and $f:(0,+\infty) \rightarrow[0,+\infty)$ be the function defined by: $f(t)=t^{-a}-1$ if $t \in(0,1]$ and $f(t)=0$ if $t>1$ and $g:(0,+\infty) \rightarrow[0,+\infty)$ be the function defined by: $g(t)=1$ if $t \in(0,1]$ and $g(t)=t^{-a}$ if $t>1$.

Let $\mathcal{M}, \tau$ and $P$ be as in Proposition 3.13. Let $T_{1}=\int_{0}^{+\infty} f(t) d P_{t}, T_{2}=$ $\int_{0}^{+\infty} g(t) d P_{t}$. The operators $T_{1}$ and $T_{2}$ are measurable and $\mu_{t}\left(T_{1}\right)=f(t)$, $\mu_{t}\left(T_{2}\right)=g(t)$. Since $T_{1}$ is a $\tau$-finite-rank operator and $T_{2}$ is bounded, it follows from Theorem 3.12 that they are tensor admissible operators. However, $T_{1}+$ $T_{2}$ is not tensor admissible as we saw in the previous example.

\section{Tensor stability}

We start this section by recalling several well-known notions.

Definition 4.1. A linear subspace $\mathscr{S}$ of $\mathscr{L}$ is called a Calkin function space if it satisfies the following condition: for every $f \in \mathscr{S}$ and $g \in \mathscr{L}$ such that $g^{\star} \leq f^{\star}$ we have that $g \in \mathscr{S}$.

Let $\lambda>0$. We consider the dilation operator $D_{\lambda}: \mathscr{L} \rightarrow \mathscr{L}$ defined by

$$
D_{\lambda}(f)(t)=f\left(\lambda^{-1} t\right) .
$$

It follows from [9, p. 54] that if $\mathscr{S}$ is a Calkin function space, $\lambda>0$ and $f \in \mathscr{S}$, then $D_{\lambda} f \in \mathscr{S}$.

Let $\mathscr{V}$ be a linear space. Recall that a quasi-norm on $\mathscr{V}$ is a non-negative function $x \mapsto\|x\|$ defined on $\mathscr{V}$ and satisfying the same axioms as a norm except for the triangle inequality which is replaced by the requirement: There exists a constant $c>0$ such that

$$
\|x+y\| \leq c(\|x\|+\|y\|),
$$

for all $x, y \in \mathscr{V}$. 
Definition 4.2. A Calkin function space $\mathscr{E}$ is called a symmetric quasinormed function space (or a symmetric quasi-normed space) if there exists a quasi-norm $\rho$ on $\mathscr{E}$ with the following property: If $f \in \mathscr{E}, g \in \mathscr{E}$ and $f^{\star} \leq g^{\star}$, then $\rho(f) \leq \rho(g)$. If $\rho$ is a norm with this property, $\mathscr{E}$ is called a symmetric normed function space (or a symmetric normed space).

Definition 4.3. Let $(\mathcal{M}, \tau)$ be a von Neumann algebra with a faithful semi-finite normal trace $\tau$. A subspace $\mathcal{J}$ of $\overline{\mathcal{M}}$ such that for every $T \in \mathcal{J}$ and $A, B \in \mathcal{M}$ we have that $A T B \in \mathcal{J}$ is called a submodule of $\overline{\mathcal{M}}$.

Let $\mathscr{E}$ be a Calkin function space. Set $\mathscr{E}(\mathcal{M})=\left\{T \in \overline{\mathcal{M}}: \mu_{t}(T) \in \mathscr{E}\right\}$. By Proposition 2.1(v), $\mathscr{E}(\mathcal{M})$ is a submodule of $\overline{\mathcal{M}}$. It is known that, when $\mathscr{M}$ is a semi-finite factor, the submodules of $\overline{\mathcal{M}}$ are in one-to-one correspondence with the Calkin spaces contained in $\mathscr{L}$ [7]. It follows that, in this case, every submodule of $\overline{\mathcal{M}}$ is of the form $\mathscr{E}(\mathcal{M})$ for some Calkin space $\mathscr{E}$.

Let $(\mathscr{E}, \rho)$ be a symmetric quasi-normed space and $(\mathcal{M}, \tau)$ be a von Neumann algebra with a faithful semi-finite normal trace $\tau$. We define a function $\bar{\rho}: \mathscr{E}(\mathcal{M}) \rightarrow[0,+\infty)$ by

$$
\bar{\rho}(T)=\rho(\mu(T)), \quad T \in \mathscr{E}(\mathcal{M}) .
$$

It is not hard to see that $\bar{\rho}$ is a quasi-norm on $\mathscr{E}(\mathcal{M})$ (see [8]). If $(\mathscr{E}, \rho)$ is a normed space then $(\mathscr{E}(\mathcal{M}), \bar{\rho})$ is a normed space. This important result was proved by Dodds, Dodds and de Pagter [3] in the case where $\rho$ is a Fatou norm and by Kalton and Sukochev [8] in the general case. If $(\mathscr{E}, \rho)$ is a Banach space then $(\mathscr{E}(\mathcal{M}), \bar{\rho})$ is also a complete normed space $([3],[8])$. We also note that if $(\mathscr{E}, \rho)$ is a complete symmetric quasi-normed space then $(\mathscr{E}(\mathcal{M}), \bar{\rho})$ is also a complete quasi-normed space ([13]).

The following theorem is a consequence of Theorem 3.8.

TheOrem 4.4. Let $(\mathcal{M}, \tau),(\mathcal{N}, \sigma)$ be von Neumann algebras with faithful semi-finite normal traces $\tau$ and $\sigma$ acting on the Hilbert spaces $\mathcal{H}_{1}$ and $\mathcal{H}_{2}$ respectively.

(1) Let $\mathscr{E}_{1}, \mathscr{E}_{2}, \mathscr{E}_{3}$ be Calkin function spaces. Assume that for every $f \in \mathscr{E}_{1}$ and $g \in \mathscr{E}_{2}$ we have that $(f \otimes g)^{\star} \in \mathscr{E}_{3}$. Then

$$
\mathscr{E}_{1}(\mathcal{M}) \otimes \mathscr{E}_{2}(\mathcal{N}) \subseteq \mathscr{E}_{3}(\mathcal{M} \bar{\otimes} \mathcal{N}) .
$$

(2) Let $\left(\mathscr{E}_{1}, \rho_{1}\right),\left(\mathscr{E}_{2}, \rho_{2}\right),\left(\mathscr{E}_{3}, \rho_{3}\right)$ be symmetric quasi-normed spaces and $C>0$. Assume that for every $f \in \mathscr{E}_{1}$ and every $g \in \mathscr{E}_{2}$, we have $(f \otimes g)^{\star} \in$ $\mathscr{E}_{3}$ and

$$
\rho_{3}\left((f \otimes g)^{\star}\right) \leq C \rho_{1}(f) \rho_{2}(g) .
$$

Then

$$
\mathscr{E}_{1}(\mathcal{M}) \otimes \mathscr{E}_{2}(\mathcal{N}) \subseteq \mathscr{E}_{3}(\mathcal{M} \bar{\otimes} \mathcal{N})
$$

and for every $T \in \mathscr{E}_{1}(\mathcal{M})$ and $S \in \mathscr{E}_{2}(\mathcal{N})$ we have

$$
\bar{\rho}_{3}(T \otimes S) \leq C \bar{\rho}_{1}(T) \bar{\rho}_{2}(S) .
$$


We combine the above theorem and results of O'Neil on tensor products of Lorentz spaces [11] to obtain Theorem 4.5 below. We first recall the definition of Lorentz spaces $\mathscr{L}_{(p, q)}$.

Let $f \in \mathscr{L}$ and $0<p<+\infty, 0<q \leq+\infty$. We set

$$
\|f\|_{p, q}= \begin{cases}\left(\int_{0}^{+\infty}\left(f^{*}(t) t^{\frac{1}{p}}\right)^{q} \frac{d m(t)}{t}\right)^{\frac{1}{q}} & \text { if } q<+\infty \\ \sup _{t>0} t^{\frac{1}{p}} f^{*}(t) & \text { if } q=+\infty\end{cases}
$$

and

$$
\mathscr{L}_{(p, q)}=\left\{f \in \mathscr{L}:\|f\|_{p, q}<+\infty\right\}
$$

(see [11, Definition 6.5]). It is clear that the spaces $\mathscr{L}_{(p, q)}$ are Calkin spaces and it is known that they are complete symmetric quasi-normed function spaces [6, Theorem 1.4.11]. Theorem 4.5 below follows from [11, Theorem 7.7], Theorem 4.4 and [9, Theorem 2.4.4].

Theorem 4.5. Let $0<p<+\infty$ and $0<q, r, s \leq+\infty$ and let $(\mathcal{M}, \tau),(\mathcal{N}, \sigma)$ be von Neumann algebras with faithful semi-finite normal traces $\tau$ and $\sigma$ acting on the Hilbert spaces $\mathcal{H}_{1}$ and $\mathcal{H}_{2}$ respectively. A necessary and sufficient condition in order that for every $T \in \mathscr{L}_{(p, q)}(\mathcal{M})$ and $S \in \mathscr{L}_{(p, r)}(\mathcal{N})$ we have that $T \otimes S \in \mathscr{L}_{(p, s)}(\mathcal{M} \bar{\otimes} \mathcal{N})$ is that $p, q, r$, s satisfy the inequalities:

$$
q \leq s, \quad r \leq s, \quad \frac{1}{p}+\frac{1}{s} \leq \frac{1}{q}+\frac{1}{r} .
$$

In that case there exists a constant $K$ which depends only on $p, q, r, s$ such that for every $T \in \mathscr{L}_{(p, q)}(\mathcal{M})$ and $S \in \mathscr{L}_{(p, r)}(\mathcal{N})$ we have that

$$
\|\mu(T \otimes S)\|_{(p, s)} \leq K\|\mu(T)\|_{(p, q)}\|\mu(S)\|_{(p, r)} .
$$

From now on, we assume that $(\mathcal{M}, \tau)$ is a factor of type $I I_{\infty}$.

Definition 4.6. (1) A Calkin function space $\mathscr{E}$ is called tensor stable if for every $f \in \mathscr{E}$ and $g \in \mathscr{E}$ we have that $(f \otimes g)^{\star} \in \mathscr{E}$.

(2) Let $\mathscr{E}$ be a Calkin function space. We shall say that the submodule $\mathscr{E}(\mathcal{M})$ is tensor stable if $\mathscr{E}$ is a tensor stable Calkin function space.

REMARK 4.7. Let $\mathscr{E}$ be a Calkin function space. It follows from Theorem 3.8 that the submodule $\mathscr{E}(\mathcal{M})$ is tensor stable if and only if

$$
\mathscr{E}(\mathcal{M}) \otimes \mathscr{E}(\mathcal{M}) \subseteq \mathscr{E}(\mathcal{M} \otimes \mathcal{M})
$$

REMARK 4.8. Let $0<p<+\infty$ and $0<q \leq+\infty$. It follows from [11, Theorem 7.7] that the Calkin function space $\mathscr{L}_{(p, q)}$ is tensor stable if and only if $q \leq p$. It follows from Theorem 4.5 that the submodule $\mathscr{L}_{(p, q)}(\mathcal{M})$ is tensor stable if and only if $q \leq p$.

Lemma 4.9. Let $f, g \in \mathscr{D}$ and $\theta \in(0,1)$. Then there exists a constant $C>0$ such that $f \leq C g$ if and only if there exists an integer $r \geq 0$ such that for every $k \in \mathbb{Z}$ we have that

$$
A_{k}(f, \theta) \leq A_{k+r}(g, \theta) .
$$


Proof. Assume $f \leq C g$. We consider $r \in \mathbb{Z}, r \geq 0$ such that $\theta^{r} C \leq 1$. Let $x \in J_{n}(f, \theta)$. Then $f(x)>\theta^{n}$ and so $g(x) \geq C^{-1} f(x) \geq \theta^{r} f(x)>\theta^{n+r}$. It follows that $x \in J_{n+r}(g, \theta)$ and $A_{k}(f, \theta) \leq A_{k+r}(g, \theta)$.

Suppose now that there exists an integer $r \geq 0$ such that for every $k \in \mathbb{Z}$ we have that $A_{k}(f, \theta) \leq A_{k+r}(g, \theta)$. Let $n$ be such that $x \in I_{n}(f, \theta)$. Then $x \in\left[A_{n}(f, \theta), A_{n+1}(f, \theta)\right)$ and we have

$$
A_{n}(f, \theta) \leq x<A_{n+1}(f, \theta) \leq A_{n+r+1}(g, \theta)
$$

by assumption. We thus have

$$
g(x) \geq g\left(A_{n+r+1}(g, \theta)\right)>\theta^{n+r+2} .
$$

But then $f(x) \leq \theta^{n}=\theta^{n+r+2} \theta^{-r-2} \leq g\left(A_{n+r+1}(g, \theta)\right) \theta^{-r-2} \leq g(x) \theta^{-r-2}$.

Let $f \in \mathscr{D}$. Then it follows from $[9$, p. 54] that the set

$$
\left\{g \text { : there exists a } C>0 \text {, and } \lambda>0 \text { such that } g^{\star} \leq C D_{\lambda} f\right\}
$$

is a Calkin function space and it is contained in every Calkin function space that contains $f$. Hence, it is the least Calkin space containing $f$. We will denote this space by $\mathscr{S}_{f}$. We will say that a Calkin space is singly generated if it is of the form $\mathscr{S}_{f}$ for some $f \in \mathscr{D}$.

TheOREM 4.10. The Calkin space generated by $f \in \mathscr{D}$ is tensor stable if and only if there exists a constant $C>0$ and $\lambda>0$ such that $(f \otimes f)^{\star} \leq C D_{\lambda} f$.

Proof. If $\mathscr{S}_{f}$ is tensor stable, then $(f \otimes f)^{\star} \in \mathscr{S}_{f}$ and hence there exist $C>0$ and $\lambda>0$ such that $(f \otimes f)^{\star} \leq C D_{\lambda} f$.

For the converse, assume that there exist $C>0$ and $\lambda>0$ such that $(f \otimes$ $f)^{\star} \leq C D_{\lambda} f$. Let $g_{1}, g_{2} \in \mathscr{S}_{f}$. Then there exist $K>0, M>0$ and $\nu>0, \kappa>0$ such that $g_{1}^{\star} \leq K D_{\nu} f$ and $g_{2}^{\star} \leq M D_{\kappa} f$. We show that $\left(g_{1} \otimes g_{2}\right)^{\star} \in \mathscr{S}_{f}$. We have

$$
\left(g_{1} \otimes g_{2}\right)^{\star}=\left(g_{1}^{\star} \otimes g_{2}^{\star}\right)^{\star}
$$

by Corollary 3.9. Hence we may assume that $g_{1}, g_{2} \in \mathscr{D}$. Let $\xi=\max \{\nu, \kappa\}$. Then, $g_{1} \leq K D_{\nu} f \leq K D_{\xi} f$ and $g_{2} \leq M D_{\kappa} f \leq M D_{\xi} f$. By Lemma 3.4, we have

$$
\left(g_{1} \otimes g_{2}\right)^{\star} \leq K M\left(D_{\xi} f \otimes D_{\xi} f\right)^{\star} .
$$

But

$$
\left(D_{\xi} f \otimes D_{\xi} f\right)^{\star}=D_{\xi}(f \otimes f)^{\star} .
$$

Hence,

$$
\left(g_{1} \otimes g_{2}\right)^{\star} \leq K M C D_{\xi} D_{\lambda} f
$$

and $\left(g_{1} \otimes g_{2}\right)^{\star} \in \mathscr{S}_{f}$.

Corollary 4.11. Let $f \in \mathscr{D}$ and $\theta \in(0,1)$. The Calkin space $\mathscr{S}_{f}$ is tensor stable if and only if there exist an integer $r \geq 0$ and $C>0$ such that for every $k \in \mathbb{Z}$ we have

$$
A_{k}\left((f \otimes f)^{\star}, \theta\right) \leq C A_{k+r}(f, \theta) .
$$


Proof. It follows from Lemma 4.9, Proposition 4.10 and the fact that $A_{k}\left(D_{\lambda} f, \theta\right)=\lambda A_{k}(f, \theta)$.

Definition 4.12. Let $(\mathcal{M}, \tau)$ be a factor of type $I I_{\infty}$ and $\mathcal{J}$ be a submodule of $\overline{\mathcal{M}}$. We will say that $\mathcal{J}$ is singly generated if there exists $T \in \overline{\mathcal{M}}$ such that $\mathcal{J}$ is the least submodule of $\overline{\mathcal{M}}$ that contains $T$. In this case, we will say that $\mathcal{J}$ is generated by $T$.

REMARK 4.13. Let $(\mathcal{M}, \tau)$ be a factor of type $I I_{\infty}, T \in \overline{\mathcal{M}}$ and $\mathcal{J}$ the submodule generated by $T$. Let $f=\mu(T)$. Then $\mathcal{J}=\mathscr{S}_{f}(\mathcal{M})$.

Definition 4.14. Let $(\mathcal{M}, \tau)$ be a factor of type $I I_{\infty}$. A function $f \in \mathscr{D}$ will be called tensor stable if $\mathscr{S}_{f}$ is tensor stable. An operator $T \in \overline{\mathcal{M}}$ will be called tensor stable if the submodule of $\overline{\mathcal{M}}$ generated by $T$ is tensor stable.

REMARK 4.15. It follows from Remark 3.3 and Corollary 4.11 that a bounded $\tau$-finite rank operator is tensor stable.

Let $\mathcal{M}, \tau$ and $P$ be as in Proposition 3.13. Let $f \in \mathscr{D}$ and $T=$ $\int_{0}^{+\infty} f(t) d P_{t}$. The operator $T$ is measurable and $\mu_{t}(T)=f(t)$. By Remark 4.13, the operator $T$ is tensor stable (resp. bounded, admissible) if and only if $f$ is a tensor stable (resp. bounded, admissible) function. It follows that in order to construct an operator in $\overline{\mathcal{M}}$ with a certain property it is sufficient to construct a function in $\mathscr{D}$ with the corresponding property.

ExAmple 4.16 (A bounded not tensor stable operator). Let $f \in \mathscr{D}$ be the function defined by:

$$
f=\sum_{n \in \mathbb{Z}} 2^{-n} \chi_{I_{n}}
$$

where: $I_{n}=[n, n+1)$ if $n \geq 1, I_{0}=(0,1)$ and $I_{n}=\emptyset$ if $n \leq-1$. Then $a_{n}(f, 1 / 2)=m\left(I_{n}\right)=1$ for $n \geq 0$ and $a_{n}(f, 1 / 2)=0$ for $n \leq-1$. By Corollary 3.6, for $n>0$ we have

$$
A_{n}\left((f \otimes f)^{\star}, 1 / 2\right)=\sum_{i+j<n} a_{i}(f, 1 / 2) a_{j}(f, 1 / 2)=n(n+1) / 2 .
$$

Let $r \in \mathbb{Z}, r>0$. For $n>0$, we have

$$
A_{n+r}(f, 1 / 2)=\sum_{i=0}^{n+r-1} a_{i}(f, 1 / 2)=n+r .
$$

Since there are no $C>0$ and $r \in \mathbb{Z}, r>0$ such that $n(n+1) / 2 \leq C(n+r)$ for every $n$, it follows from Corollary 4.11 that $f$ is not tensor stable.

EXAMPLE 4.17 (An unbounded tensor admissible not tensor stable operator). Let $f \in \mathscr{D}$ be the function defined by:

$$
f=\sum_{n \in \mathbb{Z}} 2^{-n} \chi_{I_{n}}
$$


where $I_{n}=\left[2^{n / a}, 2^{(n+1) / a}\right)$ if $n<0$ and $I_{n}=\emptyset$ if $n \geq 0$, for some $a>0$. Then $a_{n}(f, 1 / 2)=m\left(I_{n}\right)=2^{n / a}\left(2^{1 / a}-1\right)$ if $n<0$ and $a_{n}(f, 1 / 2)=0$ if $n \geq 0$. Set $a_{n}=a_{n}(f, 1 / 2)$. It follows from Theorem 3.12 that $f$ is tensor admissible.

It follows from Corollary 3.6 that

$$
A_{n}\left((f \otimes f)^{\star}, 1 / 2\right)=\sum_{i+j<n} a_{i} a_{j}
$$

for every $n \in \mathbb{Z}$. Since for $k<-1$ we have

$$
\sum_{i+j=k} a_{i} a_{j}=\sum_{i<0, j<0, i+j=k} 2^{i / a} 2^{j / a}\left(2^{1 / a}-1\right)^{2}=(|k|-1) 2^{k / a}\left(2^{1 / a}-1\right)^{2}
$$

we obtain for $n<0$

$$
A_{n}\left((f \otimes f)^{\star}, 1 / 2\right)=\sum_{i+j<n} a_{i} a_{j}=\sum_{k=-\infty}^{n-1}(|k|-1) 2^{k / a}\left(2^{1 / a}-1\right)^{2} .
$$

Assume that there exist $r$ and $C$ such that $A_{n}\left((f \otimes f)^{\star}, 1 / 2\right) \leq A_{n+r}(f, 1 / 2)$ for every $n \in \mathbb{Z}$. If $n \leq \min \{-1,-r\}$ we obtain

$$
\begin{aligned}
\sum_{k=-\infty}^{n-1}(|k|-1) 2^{k / a}\left(2^{1 / a}-1\right)^{2} & \leq \sum_{i=-\infty}^{n+r-1} 2^{i / a}\left(2^{1 / a}-1\right) \\
& =2^{(n+r-1) / a}\left(2^{1 / a}-1\right) \frac{2^{1 / a}}{2^{1 / a}-1}=2^{(n+r) / a} .
\end{aligned}
$$

Hence

$$
\begin{gathered}
(|n-1|-1) 2^{(n-1) / a}\left(2^{1 / a}-1\right)^{2} \leq 2^{(n+r) / a} \\
\Rightarrow \quad(|n-1|-1)\left(2^{1 / a}-1\right)^{2} \leq 2^{(r+1) / a}
\end{gathered}
$$

for every $n \in \mathbb{Z}$ such that $n \leq \min \{-1,-r\}$ which is absurd. It follows from Corollary 4.11 that $f$ is not tensor stable.

EXAmPLE 4.18 (A bounded tensor stable operator). Let $a_{0}, a_{1}, \ldots$ be the sequence of Catalan numbers. They are defined as follows:

$$
a_{0}=1, \quad a_{n+1}=\sum_{i=1}^{n} a_{i} a_{n-i}
$$

We set $I_{n}=\emptyset$ for $n<0, I_{0}=(0,1)$ and $I_{n}=\left[\sum_{i=0}^{n-1} a_{i}, \sum_{i=0}^{n} a_{i}\right)$ for $n>0$.

Let $f \in \mathscr{D}$ be the function defined by:

$$
f=\sum_{n \in \mathbb{Z}} 2^{-n} \chi_{I_{n}}
$$

Then, $a_{n}(f, 1 / 2)=m\left(I_{n}\right)=a_{n}$ for $n \geq 0$ and $a_{n}(f, 1 / 2)=m\left(I_{n}\right)=0$ for $n<0$. 
Let $n \in \mathbb{Z}$. Then

$$
\begin{aligned}
A_{n}\left((f \otimes f)^{\star}, 1 / 2\right) & =\sum_{i+j<n} a_{i}(f, 1 / 2) a_{j}(f, 1 / 2)=\sum_{i \geq 0, j \geq 0, i+j<n} a_{i} a_{j} \\
& =\sum_{k=0}^{n-1} \sum_{i \geq 0, j \geq 0, i+j=k} a_{i} a_{j}=\sum_{k=0}^{n-1} a_{k+1} \leq \sum_{k=0}^{n} a_{k} \\
& =A_{n+1}(f, 1 / 2) .
\end{aligned}
$$

It follows from Corollary 4.11 that $f$ is tensor stable.

EXAMPLE 4.19. An unbounded tensor stable operator.

Let $0=n_{0}<n_{1}<n_{2}<\cdots$ a strictly increasing sequence of positive integers satisfying the following conditions:

(1) For every $k \geq 0$,

$$
\sum_{i=0}^{k} n_{i}<n_{k+1}
$$

(2) There exists $C>0$ such that

$$
n_{k+1} / n_{k} \leq C
$$

for every $k \geq 1$.

We set $N_{0}=0$ and, $N_{k}=\sum_{i=0}^{k} n_{k}$ for $k=1,2,3, \ldots$, and

$$
a_{0}=\frac{1}{2} \quad \text { and } \quad a_{i}=\frac{1}{n_{k+1}} \frac{1}{2^{k+2}}, \quad \text { if } i \in\left[N_{k}+1, N_{k+1}\right] .
$$

We also set $I_{n}=\left[1-\sum_{i=0}^{|n|} a_{i}, 1-\sum_{i=0}^{|n|-1} a_{i}\right)$ for $n<0, I_{0}=\left[1-a_{0}, 1\right)$ and $I_{n}=\emptyset$ for $n>0$.

Let $f \in \mathscr{D}$ be the function defined by: $f=\sum_{n \in \mathbb{Z}} 2^{-n} \chi_{I_{n}}=\sum_{n=-\infty}^{0} 2^{-n} \chi_{I_{n}}$. Then $a_{n}(f, 1 / 2)=a_{|n|}$ if $n \leq 0$ and $a_{n}(f, 1 / 2)=0$ if $n>0$.

Suppose that $n<0,|n| \in\left[N_{k}+1, N_{k+1}\right]$, that is $|n|=N_{k}+l$, with $1 \leq l \leq$ $n_{k+1}$.

We calculate $a_{n}\left((f \otimes f)^{\star}, 1 / 2\right)$. By Corollary 3.6, we have

$$
\begin{aligned}
a_{n}\left((f \otimes f)^{\star}, 1 / 2\right) & =\sum_{i+j=n} a_{i}(f, 1 / 2) a_{j}(f, 1 / 2) \\
& =\sum_{i+j=n} a_{|i|} a_{|j|}=\sum_{|i|+|j|=|n|} a_{|i|} a_{|j|} .
\end{aligned}
$$

Suppose that $|i|+|j|=|n|$; we claim that $|i|>N_{k-1}$ or $|j|>N_{k-1}$. Indeed, if $|i| \leq N_{k-1}$ and $|j| \leq N_{k-1}$, then, by (1) above, $|i|+|j| \leq 2 N_{k-1}<N_{k}+1$. This is a contradiction since $|i|+|j|=|n| \in\left[N_{k}+1, N_{k+1}\right]$. Set $I=\{|i| \leq$ $\left.N_{k-1}\right\}, J=\left\{|i|>N_{k-1}\right\}$. We have

$$
a_{n}\left((f \otimes f)^{\star}, 1 / 2\right)=\sum_{|i|+|j|=|n|} a_{|i|} a_{|j|}=\sum_{|i| \in I} a_{|i|} a_{|n|-|i|}+\sum_{|i| \in J} a_{|i|} a_{|n|-|i|} .
$$


If $|i| \in I$, then $|n|-|i|=|j|>N_{k-1}$ and

$$
a_{|n|-|i|} \leq \frac{1}{n_{k}} \frac{1}{2^{k+1}}
$$

Since $\sum_{i \in \mathbb{Z}} a_{i}=1$ we obtain

$$
\sum_{|i| \in I} a_{|i|} a_{|n|-|i|} \leq \frac{1}{n_{k}} \frac{1}{2^{k+1}} .
$$

If $|i| \in J$, then $|i|>N_{k-1}$ and

$$
a_{|i|} \leq \frac{1}{n_{k}} \frac{1}{2^{k+1}} .
$$

Since $\sum_{i \in \mathbb{Z}} a_{i}=1$ we obtain

$$
\sum_{|i| \in J} a_{|i|} a_{|n|-|i|} \leq \frac{1}{n_{k}} \frac{1}{2^{k+1}} .
$$

Hence,

$$
a_{n}\left((f \otimes f)^{\star}, 1 / 2\right) \leq 2 \frac{1}{n_{k}} \frac{1}{2^{k+1}} \leq 4 C \frac{1}{n_{k+1}} \frac{1}{2^{k+2}}=4 C a_{n}(f, 1 / 2) .
$$

For $n=0$, we have

$$
a_{0}\left((f \otimes f)^{\star}, 1 / 2\right)=a_{0}^{2} \leq 4 C a_{0}=4 C a_{0}(f, 1 / 2)
$$

since $C>1$. It follows from Corollary 4.11 that $f$ is tensor stable.

\section{REFERENCES}

[1] M. Anoussis, V. Felouzis and I. G. Todorov, s-numbers of elementary operators on $C^{*}$-algebras, J. Operator Theory 66 (2011), 235-260. MR 2844465

[2] C. Bennett and R. Sharpley, Interpolation of operators, Pure and Applied Mathematics, vol. 129, Academic Press, Boston, MA, 1988. MR 0928802

[3] P. G. Dodds, T. K. Dodds and B. de Pagter, Non-commutative Banach function spaces, Math. Z. 201 (1989), 583-597. DOI:10.1007/BF01215160. MR 1004176

[4] T. Fack, Sums of commutators in non-commutative Banach function spaces, J. Funct. Anal. 207 (2004), 358-398. DOI:10.1016/S0022-1236(03)00235-0. MR 2032994

[5] T. Fack and H. Kosaki, Generalized s-numbers of $\tau$-compact operators, Pacific J. Math. 123 (1986), 269-300. MR 0840845

[6] L. Grafakos, Classical Fourier analysis, 2nd ed., Graduate Texts in Mathematics, vol. 249, Springer, New York, 2008. MR 2445437

[7] D. Guido and T. Isola, Singular traces on semifinite von Neumann algebras, J. Funct. Anal. 134 (1995), 451-485. DOI:10.1006/jfan.1995.1153. MR 1363808

[8] N. Kalton and F. Sukochev, Symmetric norms and spaces of operators, J. Reine Angew. Math. 621 (2008), 81-121. DOI:10.1515/CRELLE. 2008.059. MR 2431251

[9] S. Lord, F. Sukochev and D. Zanin, Singular traces, theory and applications, De Gruyter Studies in Mathematics, vol. 46, De Gruyter, Berlin, 2013. MR 3099777

[10] E. Nelson, Notes on non-commutative integration, J. Funct. Anal. 15 (1974), 103-116. MR 0355628

[11] R. O'Neil, Integral transforms and tensor products on Orlicz spaces and $L(p, q)$ spaces, J. Anal. Math. 21 (1968), 1-276. MR 0626853 
[12] W. F. Stinespring, Integration theorems for gages and duality for unimodular groups, Trans. Amer. Math. Soc. 90 (1959), 15-56. MR 0102761

[13] F. Sukochev, Completeness of quasi-normed symmetric operator spaces, Indag. Math. (N.S.) 25 (2014), 376-388. DOI:10.1016/j.indag.2012.05.007. MR 3151823

[14] G. Weiss, Classification of certain commutar ideals and the tensor product closure property, Integral Equations Operator Theory 12 (1989), 99-128. DOI:10.1007/BF01199759. MR 0973049

M. Anoussis, Department of Mathematics, University of the Aegean, 83200 Karlovasi, Samos, Greece

E-mail address: mano@aegean.gr

V. Felouzis, Department of Mathematics, University of the Aegean, 83200 Karlovasi, Samos, Greece

E-mail address: felouzis@aegean.gr

I. G. Todorov, Pure Mathematics Research Centre, Queen's University Belfast, Belfast BT7 1NN, United Kingdom

E-mail address: i.todorov@qub.ac.uk 